\title{
Las relaciones internacionales en la novela gráfica. Una aproximación
}

\author{
David Fernández de Arriba
}

Licenciado en Humanidades por la Universitat Pompeu Fabra en la especialidad de Historia, realizó un Posgrado en Sociedades Africanas y Desarrollo en el mismo centro. Profesor de educación secundaria desde el año 2010, ha impartido diversas asignaturas en los campos de las ciencias sociales y de las lenguas, tratando de incluir el cómic como herramienta didáctica. En septiembre de 2014 creó el blog sobre cómics históricos Historia y cómic. Aprender historia leyendo cómics, en el que ha reseñado un centenar de obras y ha creado una decena de materiales didácticos para el uso del cómic en el aula. Escribe sobre cómic en la Revista Endavant y recientemente se ha convertido en miembro de la Asociación de Críticos y Divulgadores de Cómic de España.

Fecha de recepción: 4 de mayo de 2016

Fecha de aceptación definitiva: 17 de mayo de 2016 


\title{
Resumen
}

El presente ensayo trata de analizar el binomio formado por la novela gráfica y las relaciones internacionales. Tras el final de la Guerra Fría, la geopolítica mundial ha generado multitud de escenarios complejos que han provocado un aumento del interés en las relaciones internacionales. El cómic, del mismo modo que otras manifestaciones culturales, ha sido partícipe de este fenómeno. El objetivo principal de este texto es estudiar las diversas formas en las que los y las dibujantes han representado la realidad sociopolítica de diversos países alejados cultural e históricamente de Occidente.

Palabras clave: Cómic, Relaciones Internacionales, Historia, novela gráfica, guerra, Geopolítica.

\begin{abstract}
This essay tries to analyze the pairing formed by Graphic Novel and International Relations. After the end of Cold War, global geopolitics has created a lot of complex scenarios which have generated an increase on the interest in International Relations. Comic, as other cultural expressions, has been part of this trend. The main objective of this text is to study how comic artists have represented the sociopolitical and historical situation of countries far away from the West.
\end{abstract}

Key Words: Comic, International Relations, History, Graphic Novel, War, Geopolitics.

\section{Cita bibliográfica}

Fernández de Arriba, D. «Las relaciones internacionales en la novela gráfica. Una aproximación», en CuCo, Cuadernos de cómic, n. ${ }^{\circ} 6$ (2016), pp. 62-79. 
La novela gráfica nos ha acercado a multitud de contextos internacionales. De la mano de una gran variedad de autores y autoras y mediante enfoques muy diversos, el lenguaje del cómic nos ha mostrado en los últimos años la realidad de lugares y contextos muy dispares. $\mathrm{El}$ objetivo de este ensayo es analizar las formas en que se ha producido este acercamiento entre la novela gráfica y las relaciones internacionales.

\section{El marco histórico}

El hundimiento de la URSS en 1991 puso fin al periodo que el historiador británico Eric J. Hobsbawm denominó, en su canónica Historia del siglo XX, ${ }^{1}$ «el siglo xx corto», que abarcaba desde el inicio de la Primera Guerra Mundial hasta el final de la Guerra Fría, marcado por la desaparición del gigante soviético. En Occidente la sensación era de victoria y las declaraciones del presidente George Bush así lo atestiguaban:

De estos tiempos de dificultades puede emerger nuestro quinto objetivo: un nuevo orden internacional, una nueva era libre de la amenaza del terror, más decidida en la consecución de la justicia y más segura en la búsqueda de la paz. Una era en la que las naciones del mundo, del Este y del Oeste, del Norte y del Sur, puedan prosperar y vivir en armonía. ${ }^{2}$

Adquirió una gran celebridad Francis Fukuyama con su obra El fin de la historia, en la que pregonaba que el liberalismo económico y la democracia de corte occidental habían triunfado y que a partir de entonces «la economía sustituiría a las ideologías y la ciencia determinaría el discurrir de la historia». ${ }^{3}$ Todo hacía indicar que estaba surgiendo un mundo unipolar en el que la hegemonía de EE. UU. y sus aliados iba a ser indiscutida. Pero los designios de la historia se iban a empeñar en demostrar lo contrario, ya en la misma década de los noventa.

Sirvan como evidencia de lo erróneo de la tesis de Fukuyama estas palabras de Josep Fontana haciendo balance de los setenta años que habían pasado desde el final de la Segunda

${ }^{1}$ Нов sbawm, E. J. Historia del siglo XX. Barcelona, Ed. Crítica, 2007, p. 7.

2 Veiga, F. El desequilibrio como orden. Una historia de la posguerra fría. Madrid, Alianza Editorial, 2015, pp. 61-62.

3 Ibid., p. 69. 
Guerra Mundial en la introducción a su monumental Por el bien del imperio. Una historia del mundo desde 1945:

No hay paz - Irak, Afganistán, Libia o Darfur dan testimonio de ello-, la extensión de la democracia es poco más que una apariencia, y en algunos lugares ni siquiera esto, como lo demuestra la frecuencia con la que los derechos humanos son vulnerados, [...] Y lejos de la prosperidad global que se nos anunciaba, vivimos en un mundo más desigual, puesto que la divergencia entre los niveles de vida en los países desarrollados y los de aquellos que se acostumbraba a denominar «en vías de desarrollo» $[\ldots]$ sigue aumentando día a día. ${ }^{4}$

Haciendo un breve repaso a los conflictos armados que tuvieron lugar en la última década del siglo pasado y lo que llevamos de siglo xxi se observa como el final del orden bipolar no provocó una disminución de su número. Tras el final de la Segunda Guerra Mundial y la inmediata posguerra, Europa había vivido más de cuarenta años de relativa paz, pero las guerras balcánicas pusieron fin a esta situación. África había sufrido en carne propia el enfrentamiento entre las dos superpotencias - Angola, Mozambique, Etiopía, Somalia...pero el final de la Guerra Fría no supuso una mejora. El genocidio de Ruanda o la guerra del Congo, entre otros muchos estallidos violentos, así lo confirmaron. Oriente Medio, zona siempre de mucha tensión, vio como esta se acrecentaba en diversos frentes: el conflicto entre Israel y Palestina, la Guerra del Golfo, las posteriores invasiones de Afganistán e Irak...
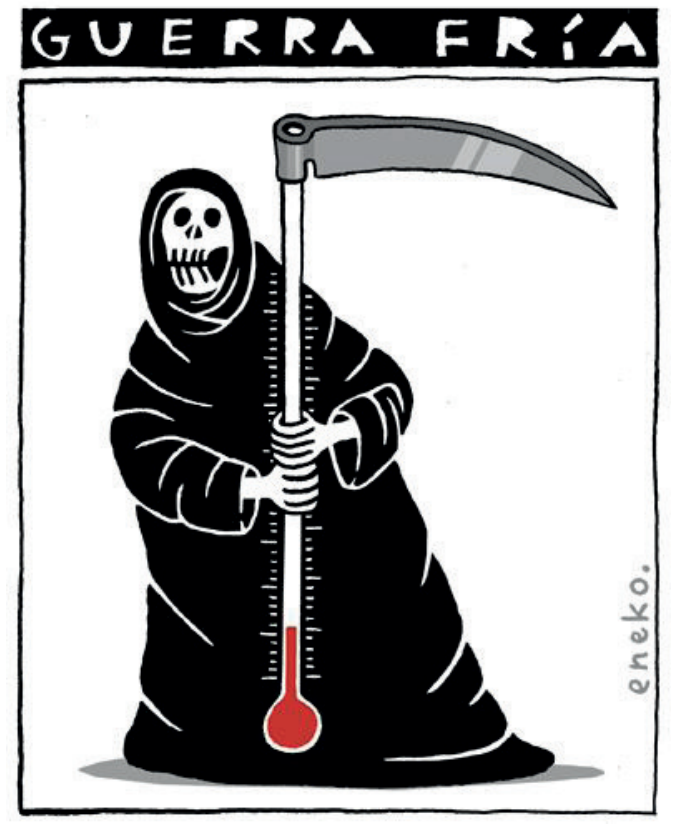

FIG 1. Enеко. «Guerra fría», en 20 minutos (5 de septiembre de 2014). Disponible online en http://blogs.20minutos.es/eneko/2014/09/05/ guerra-fria/

${ }^{4}$ Fontana, J. Por el bien del imperio. Una historia del mundo desde 1945. Barcelona, Pasado \& Presente, 2013, p. 9. 
Al mismo tiempo un nuevo fenómeno había llegado para quedarse y para cambiar la vida de buena parte de la población del planeta: la globalización. En el ámbito de las relaciones internacionales, la globalización está marcada por tres aspectos fundamentales: la mundialización, la complejidad y la interdependencia. ${ }^{5}$ En un mundo cada vez más interconectado, las consecuencias de cualquier crisis o conflicto tienen ámbito mundial. Baste señalar un par de ejemplos: la crisis financiera de 2008 que se inició en los EE. UU. y cuyos efectos se hicieron notar en el conjunto de la economía mundial; o la guerra civil siria que está poniendo en evidencia a instituciones y gobiernos europeos en sus formas de tratar a los refugiados que huyen de la violencia.

Con el fin de siglo también se generalizó el uso de Internet, hecho fundamental para comprender el mundo en que vivimos. Su rápida evolución y su cada vez mayor accesibilidad han provocado cambios muy profundos en la forma de comunicarnos y en elementos tan comunes en nuestras vidas cotidianas como los medios de comunicación. Las redes sociales han amplificado aún más estos cambios. ¿Por qué esperar a mañana a leer una noticia si puedo acceder ahora mismo a un periódico digital? ¿Por qué leer un periódico si puedo seguir en Twitter a alguien que está en el lugar de la noticia y explica con inmediatez lo que está sucediendo? ¿Por qué limitar mi interés a noticias locales o nacionales si tengo acceso a las de prácticamente todo el mundo?

\section{El auge de las Relaciones Internacionales}

Un mundo cada vez más interconectado ha aumentado el interés por conocer lo que pasa más allá de las respectivas fronteras nacionales. El estudio de las relaciones internacionales es una disciplina joven en España, pero como afirma Juan Carlos Pereira, catedrático de Historia Contemporánea en la Universidad Complutense de Madrid y presidente de la Comisión Española de Historia de la Relaciones Internacionales (CEHRI), «La Historia de las Relaciones Internacionales, como disciplina académica y como área de investigación, está ya plenamente consolidada en España». ${ }^{6}$ Así lo atestigua la creciente oferta de grados y másteres en la citada disciplina. ${ }^{7}$

Más allá del ámbito académico, esencial pero al fin y al cabo, minoritario, otros sectores también dan fe del creciente interés que despiertan las relaciones internacionales. En los últimos tiempos han surgido iniciativas como la Revista $5 \mathrm{~W}$, creada por fotoperiodistas

${ }_{5}^{5}$ Calduch, R. «Procesos de cooperación y conflicto en el sistema internacional del siglo XXI», en Pereira, J. C. (coord.) Historia de las relaciones internacionales contemporáneas. Barcelona, Ariel, 2013, pp. 701-702.

6 Pereira, J. C. (coord.) Historia de las relaciones internacionales contemporáneas. Barcelona, Ariel, 2013, p. Xx.

7 SÁenz, J. M. «Los estudios de Relaciones Internacionales en España: una historia breve y de éxito». Disponible online en http://www.esglobal.org/los-estudios-de-relaciones-internacionales-en-espana-una-historia-breve-y-de-exito/ y Rubio, D. "Los estudios en Relaciones Internacionales en España: llegada, consolidación y futuro». Disponible online en http://danielrubiosanchez.com/2015/05/08/relaciones-internacionales-y-eso-que-es/ 
y reporteros de guerra; ${ }^{8}$ los encuentros Beers and World, que se celebran regularmente en Barcelona para tratar temas de actualidad internacional; ${ }^{9}$ y otros proyectos como Passim, ${ }^{10}$ Es Global ${ }^{11} \mathrm{o}$ AfricaYe. ${ }^{12}$ Todos ellos conviven, interaccionan y se nutren con Think tanks como $\mathrm{CIDOB}^{13}$ o grupos de investigación como el Centre d'Estudis Africans ${ }^{14}$ o EurasiaNet. ${ }^{15}$

Muy alejadas ya del mundo universitario, pero con un alcance mucho mayor, se encuentran las series televisivas. En la última década las ficciones televisivas se han convertido, aún más si cabe, en un producto de consumo masivo en un mercado prácticamente de ámbito mundial. Ficciones sobre todo norteamericanas, pero también británicas, escandinavas, latinoamericanas y, en menor proporción, de otras zonas geográficas han llegado a las pantallas de millones de telespectadores de todo el mundo. Centradas en multitud de aspectos, también han aparecido, en cada vez mayor número, series que tratan elementos centrales de las relaciones internacionales.

Sin la intención de ser exhaustivo, son destacables El ala oeste de la Casa Blanca (The West Wing), sobre la política norteamericana, con muchos episodios dedicados a la política internacional; Borgen, que mantiene la misma premisa, pero en esta ocasión con el epicentro en Dinamarca; Homeland, ya directamente centrada en el espionaje, el terrorismo y con un papel central de Oriente Medio; The honourable woman, en el contexto del conflicto entre Israel y Palestina; The Americans, enfocada en la Guerra Fría y el mundo del espionaje; Deutschland 83, sobre la situación de las dos Alemanias; o House of Cards, que trata con cinismo los entresijos de la política en EE. UU.

\section{Las relaciones internacionales y el cómic}

El cómic, como todo producto cultural, es un medio dinámico y también se ha imbuido del creciente interés por la política internacional y la historia de las relaciones internacionales. El ámbito de estudio es amplísimo y, por tanto, imposible de analizar en profundidad en este breve ensayo. Las siguientes páginas se centran tan solo en la novela gráfica, es decir, en los últimos veinticinco años aproximadamente, y el objetivo es establecer las diversas maneras en que los autores se han acercado a esta temática. Sería interesantísimo, por ejemplo, poder incluir el papel que jugaron los superhéroes durante la Guerra Fría o los nuevos

\footnotetext{
8 Disponible online en http://www.revista5w.com/

9 Disponible online en http://beersandworld.com/

${ }^{10}$ Disponible online en http://www.passim.eu/

11 Disponible online en http://www.esglobal.org/

12 Disponible online en http://www.africaye.org/

13 Disponible online en http://www.cidob.org/

${ }^{14}$ Disponible online en http://centredestudisafricans.org/

15 Disponible online en http://eurasianet.es/
} 
autores árabes que están tratando la actualidad de sus países a través del cómic, pero ambos temas quedan más allá del objeto de estudio de este texto.

Se podría establecer una clasificación geográfica y tratar de catalogar las obras que han tratado aspectos centrales en los diversos conflictos o las diversas áreas geopolíticas, no obstante, creo que puede resultar de mayor interés analizar qué mecanismos han utilizado los y las dibujantes para introducirnos en la historia más reciente. Así, establezco una clasificación en tres categorías:

\subsection{Ensayo bistórico}

El ensayo, junto con la biografía, es el género predominante en los textos históricos. En el caso del cómic, han sido diversos los autores que han tratado de unir la profundidad analítica de este tipo de textos con la expresividad y la fuerza narrativa del noveno arte. Generalmente, la colaboración de un experto en la materia y un dibujante ha permitido combinar las virtudes de ambos formatos.

Un buen ejemplo de este tipo de cómics es Los mejores enemigos ${ }^{16}$ del profesor de Ciencias Políticas Jean-Pierre Filiu y el historietista David B. El objetivo de la obra es claro, como muestra su subtítulo Una historia de las relaciones entre EE. UU. y Oriente Medio. El cómic, del que se han publicado hasta el momento las dos primeras partes, abarca el periodo comprendido entre 1783 y 1953 en el primer volumen, y entre 1953 y 1984 en el segundo, aunque la idea de los autores es llegar hasta el presente.

En sus páginas se combinan a la perfección la documentada explicación histórica de Filiu con la imaginativa expresión artística de David B. Las metáforas visuales y los juegos con el blanco y negro del dibujante ilustran a la perfección los diversos episodios históricos: desde el primer choque entre el nuevo país americano y los piratas berberiscos, a finales del siglo XVIII, hasta la sangrienta guerra civil libanesa, en los años ochenta del siglo xx. La capacidad didáctica propia del cómic consigue que en 256 páginas - 128 en cada volumen- los autores sean capaces de relatar con gran acierto un gran número de conflictos y momentos trascendentes para comprender el mundo actual.

Otros ejemplos de ensayos históricos en formato cómic, que además tienen un enfoque similar, son Adictos a la guerra, ${ }^{17}$ de Joel Andreas y Una historia popular del Imperio Americano, ${ }^{18}$ adaptación de la obra de Howard Zinn La otra historia de los EE. UU. ${ }^{19}$ Ambos

\footnotetext{
${ }_{16}$ Filiu, J. P. y David B. Los mejores enemigos. Una historia de las relaciones entre EE. UU. y Oriente Medio. Barcelona, Norma Editorial, 2012 y 2015. (2 volúmenes)

17 Andreas, J. Adictos a la guerra. Por qué EE. UU. está enganchado al militarismo. Bilbao, Astiberri, 2004.

18 ZINN, H. Una bistoria popular del Imperio Americano. Madrid, Sins Entido, 2010.

19 ZINN, H. La otra bistoria de los EE. UU. Hondarribia, Hiru, 1997.
} 


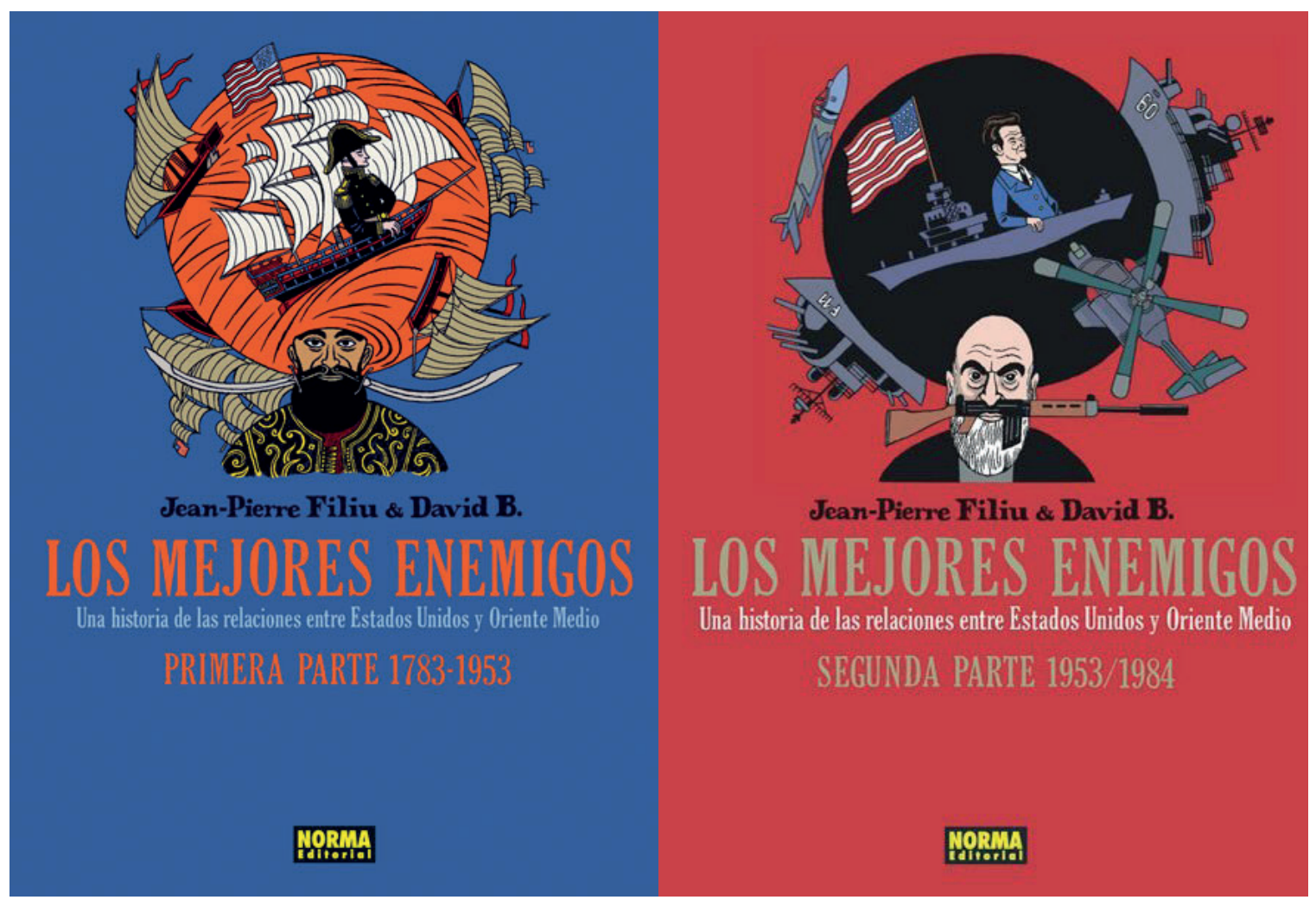

FIG 2. Filiu, J. P. y David B. Los mejores enemigos. Una historia de las relaciones entre EE. UU. y Oriente Medio. Barcelona, Norma Editorial, 2012 y 2015. Portadas de los dos volúmenes publicados en España.

títulos tratan de invalidar la versión oficial de la historia de los EE. UU., y como explican sus autores, la elección del cómic como medio no es casual. El objetivo principal es conseguir la mayor difusión posible y para ello muestran una total confianza en las posibilidades divulgativas de las viñetas.

La obra de Andreas intenta demostrar la influencia del lobby armamentístico en las decisiones en política internacional de las diferentes administraciones americanas. Para conseguirlo utiliza personajes ficticios, una madre y su hijo, que empiezan a investigar y a descubrir en qué se gasta buena parte de su presupuesto la administración norteamericana. La inclusión de personajes históricos reales consigue dotar de realismo a la sencilla trama, y al mismo tiempo, el dibujo caricaturesco intenta hacer más llevadera la profusión de datos que incluye el autor. El tono general del cómic es didáctico e intenta ser un toque de atención para la sociedad estadounidense, ya que como indica el autor en el prólogo: «Espero que este libro incite a la reflexión y el debate sobre el militarismo, y que anime a la acción positiva para cambiar el rumbo que hemos tomado.» ${ }^{20}$

${ }^{20}$ Andreas, J. Prólogo del autor a la edición de 2004. 


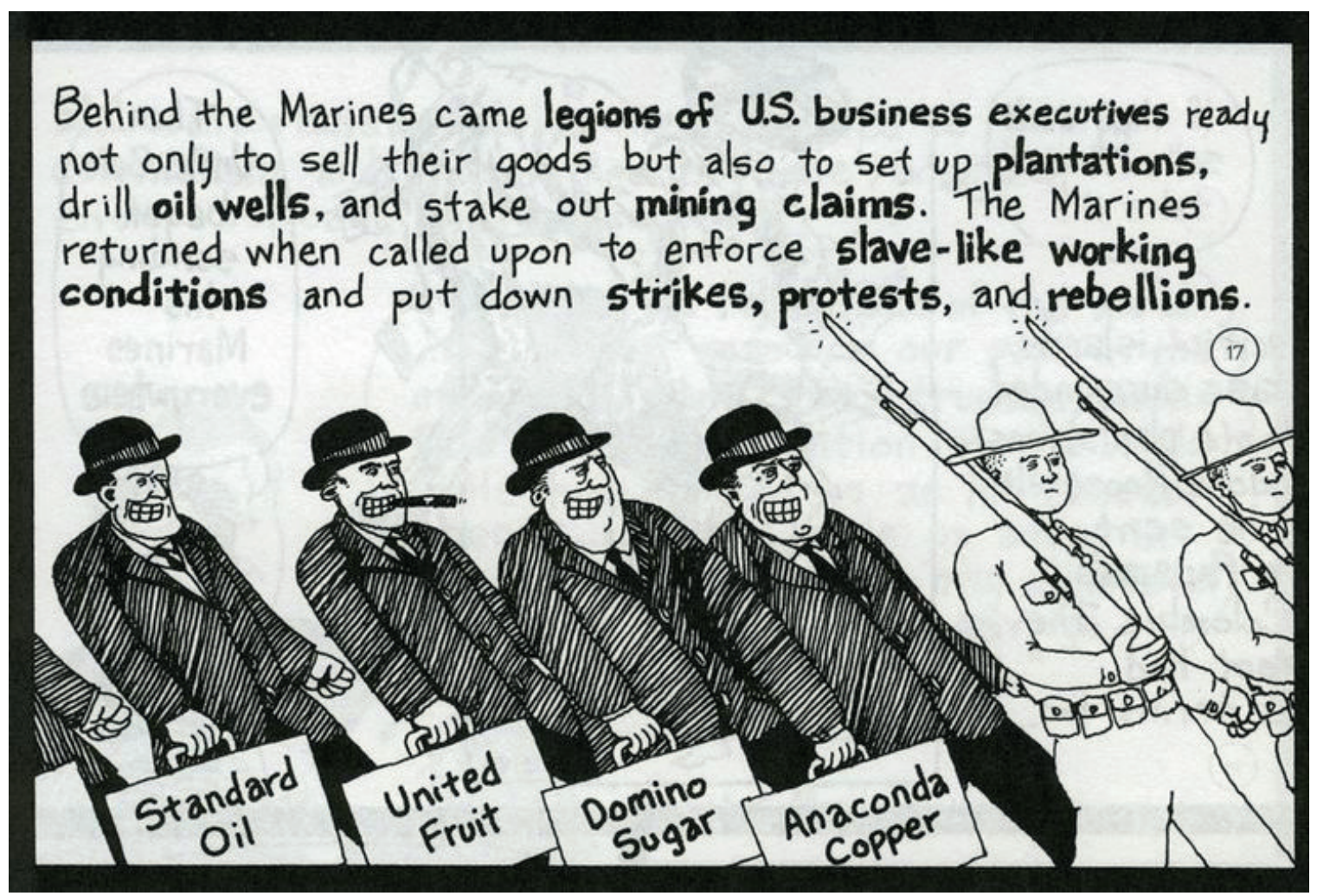

FIG 3. Andreas, J. Adictos a la guerra. Por qué EE. UU. está enganchado al militarismo. Bilbao, Astiberri, 2004. Viñeta de la página 16 en que se aprecia el tono de la obra.

En el caso de Una historia popular del Imperio Americano, a pesar de que el enfoque general es similar, Howard Zinn y sus colaboradores Mike Konopacki y Paul Buhle decidieron utilizar otros recursos. Dada la popularidad del profesor Zinn, optaron por incluirlo como un personaje central en el relato, ya que es él quien va introduciendo los diversos capítulos de la obra. Sus intervenciones, en forma de bocadillos, permiten contextualizar los episodios que son narrados mediante viñetas. La adaptación de una obra teórica de más de seiscientas páginas a un cómic de menos de trescientas debió ser complicada, y este hecho se hace notar en determinadas ocasiones en el que el ritmo narrativo se hace espeso. Pese a todo, este cómic vuelve a mostrar la capacidad sintética y divulgativa del cómic, ya que es una lectura muy enriquecedora.

\subsection{Autobiografía}

El género autobiográfico ha tenido una importancia crucial en el desarrollo del cómic de los últimos cincuenta años. En el caso que nos ocupa, esta tipología ha tenido una gran presencia y este artículo sería imposible de realizar si multitud de autores y autoras no hubieran utilizado sus propias experiencias para narrar lo que ocurría en diversos lugares del mundo. 
Debido a la gran cantidad de obras que se podrían incluir en esta categoría, he optado por dividirlas en dos subcategorías en función de la perspectiva que ofrecen los y las dibujantes.

\subsubsection{Observador interno}

Diversos han sido los dibujantes que se han acercado a la realidad de sus propios países pensando en la acogida que tendrían sus obras en Occidente. El ejemplo paradigmático de este tipo de cómic autobiográfico que explora la realidad histórica o social de un país alejado culturalmente del mundo occidental es Persépolis. ${ }^{21}$ El gran éxito de la novela gráfica de Marjane Satrapi, publicada originalmente en Francia, tuvo una gran influencia sobre multitud de autores. El mundo árabe ha tenido una gran presencia, de la mano especialmente de dibujantes que, como en el caso de Riad Sattouf, ya habían nacido en algún país europeo o de otros, como Zeina Abirached o la propia Satrapi, que se habían instalado en Europa durante su juventud.
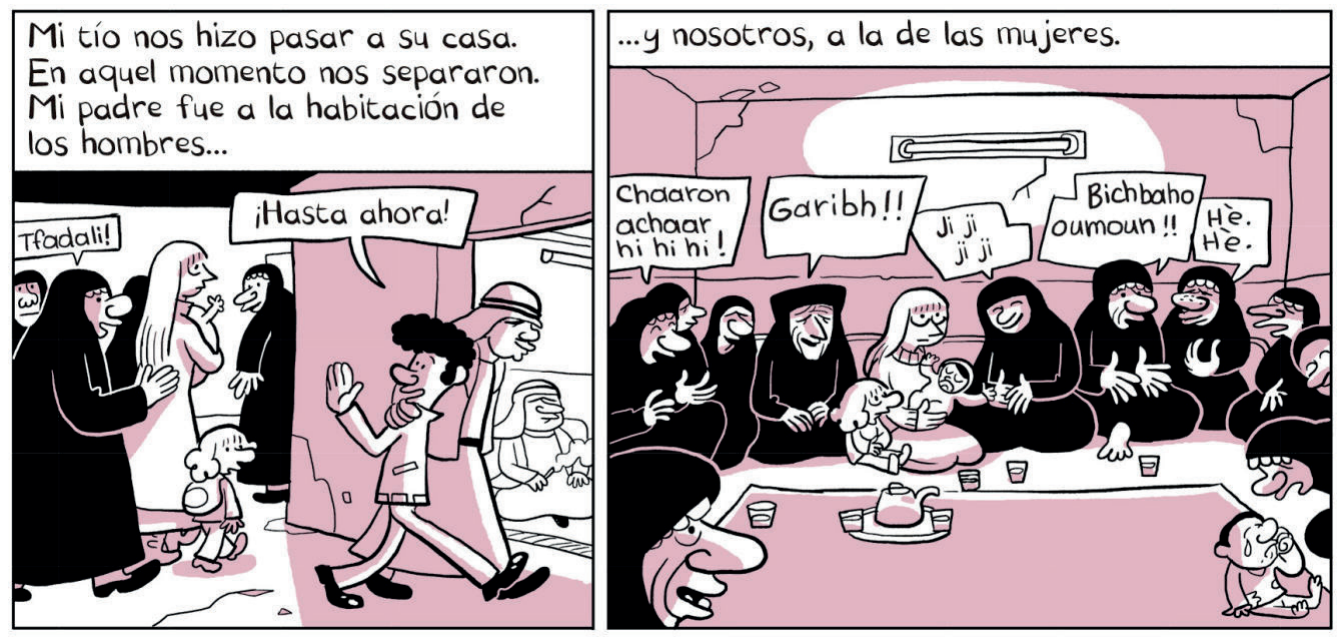

FIG 4. Sattouf, R. El árabe del futuro. Una juventud en Oriente Medio (1978-1984). Barcelona, Salamandra Graphic, 2015. Viñetas de la página 76 en las que se observa la llegada del pequeño Riad a la casa de sus familiares sirios.

Los citados autores utilizan estrategias similares para acercarnos a la realidad de sus países de origen. Tanto en Persépolis, como en El árabe del futuro ${ }^{22}$ — del que recientemente se ha publicado el segundo volumen- o en El juego de las golondrinas ${ }^{23}$ sus autores utilizan su propia visión infantil como elemento narrativo. Esta técnica permite establecer un cierto

${ }^{21}$ Satrapi, M. Persépolis. Barcelona, Norma Editorial, 2006.

${ }^{22}$ Sattouf, R. El árabe del futuro. Una juventud en Oriente Medio (1978-1984). Barcelona, Salamandra Graphic, 2015.

23 Abirached, Z. El juego de las golondrinas. Madrid, Sins Entido, 2008. 
alejamiento de los hechos, de modo que el contraste entre lo que sintieron mientras sucedían los acontecimientos que narran y sus posteriores reflexiones ya adultas consiguen transmitir un gran caudal de información.

A pesar de que los países y las situaciones que describen son muy diferentes entre sí -el Irán de la Revolución islámica en Persépolis, el Beirut de la guerra civil libanesa en El juego de las golondrinas y la Libia de Gadafi y la Siria de Hafez el Asad en El árabe del futuro-, los tres cómics tienen muchos elementos en común. El costumbrismo tiene un papel central, la presencia de sus familias tiene un gran peso y, gracias al contrapunto infantil, el sentido del humor también tiene su espacio.

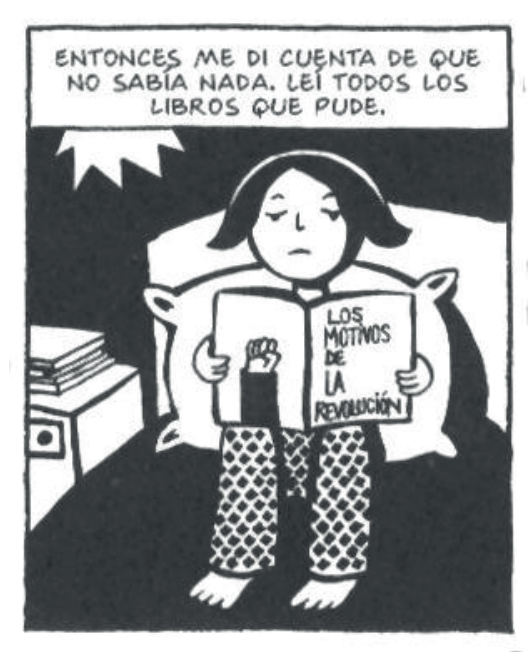

FIG. 5. Satrapi, M. Persépolis. Barcelona, Norma Editorial, 2006. Viñeta de la página 38 en que se aprecia el contraste entre la visión infantil del momento y la reflexión adulta de la autora.

Encuadrado también entre las obras que presentan un observador interno, que trata de dar a conocer la realidad sociopolítica de su país, se encuentra Nikolai Maslov. El autor ruso, utilizando en esta ocasión historias cortas, dio a conocer en Los hijos de octubre ${ }^{24}$ la situación de su Siberia natal. Maslov, tras residir durante bastantes años en Moscú, volvió a su hogar y se encontró con los efectos de la caída de la Unión Soviética. El alcoholismo, la miseria y la emigración causaban estragos y el dibujante siberiano recreó mediante sus dibujos a lápiz la melancolía y la tristeza que imperaban en su región. Maslov no utiliza grandes artificios, pero tiene éxito en presentarnos la vida de los habitantes de una de las zonas más desoladas del planeta.

Otros cómics que muestran las vivencias de sus autores y permiten analizar la situación de sus respectivos países en momentos trascendentes de su historia son:

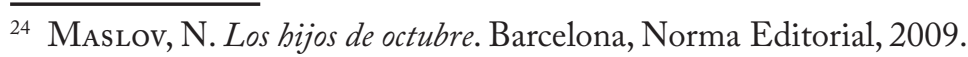


- Una vida en China, de Li Kunwu y P. Otié (Astiberri, 2010), con el que recorremos buena parte del siglo xx en el gran país asiático.

- Una guerrita de nada, de Marcelino Truong (Spaceman Books, 2015), en que accedemos a una visión de la guerra de Vietnam muy alejada de los tópicos hollywoodienses.

- Arenas movedizas, de Max Monch, Alexander Lahl y Kitty Kahane (Impedimenta, 2015), que aunque no es estrictamente autobiográfico, buena parte de lo que nos cuenta está basado en las experiencias personales de los autores en la Alemania dividida de los años ochenta.

- Patria, de Nina Bunjevac (Turner, 2015), que reconstruye su historia familiar en la antigua Yugoslavia y nos narra su propia experiencia entre la emigración en Canadá y el país balcánico gobernado por Tito.

\subsubsection{Observador externo}

Un enfoque muy diferente, pero igualmente interesante, es el de los y las autoras que viajan a lugares generalmente conflictivos para reflejar en sus cómics las realidades con las que se encuentran. Uno de los elementos esenciales de este tipo de obras es el contraste entre la visión occidental de los autores y los contextos en que se sitúan sus relatos. En los últimos veinticinco años han sido muchísimos los que han optado por llevar a cabo este tipo de novelas gráficas, con resultados dispares. Entre los que han creado obras de gran valor, un nombre destaca por encima del resto: Joe Sacco. No me extenderé sobre la trascendencia y la repercusión de la obra del autor norteamericano, ${ }^{25}$ pero vale la pena destacar que fue uno de los primeros en apostar por el cómic periodístico y se convirtió en el primer reportero de guerra que elaboraba sus reportajes en forma de cómic.

Sus obras más reconocidas son las que narran el conflicto entre Israel y Palestina - Palestina ${ }^{26}$ y Notas al pie de Gaza- ${ }^{27}$ y las que se sitúan en la guerra de Bosnia - Gorazde. Zona protegida - ${ }^{28}$ aunque en obras posteriores, muchas de ellas para prensa, se ha acercado a multitud de escenarios: desde Chechenia, la India o Malta hasta el Tribunal Penal Internacional de La Haya. Todas sus obras tienen ciertas características comunes: la inclusión de sí mismo como personaje, la intención de dar voz a todas las partes presentes en el conflicto, una gran labor de documentación o la reflexión en torno a la objetividad y la labor del periodista.

25 A este respecto es muy recomendable la lectura de Espiña Barros, D. «Apuntes a notas al pie de Gaza. El cómic periodístico de Joe Sacco», en Cuco, Cuadernos de Cómic, n. 2 (2014), pp. 92-108. Disponible online en http://cuadernosdecomic.com/docs/revista2/Apuntes a Notas al pie Gaza cuco2.pdf

${ }^{26}$ SAcco, J. Palestina. En la franja de Gaza. Barcelona, Planeta de Agostini, 2002.

27 SAcco, J. Notas al pie de Gaza. Barcelona, Reservoir Books, 2010.

${ }^{28}$ SAcco, J. Gorazde. Zona protegida. Barcelona, Planeta de Agostini, 2001. 


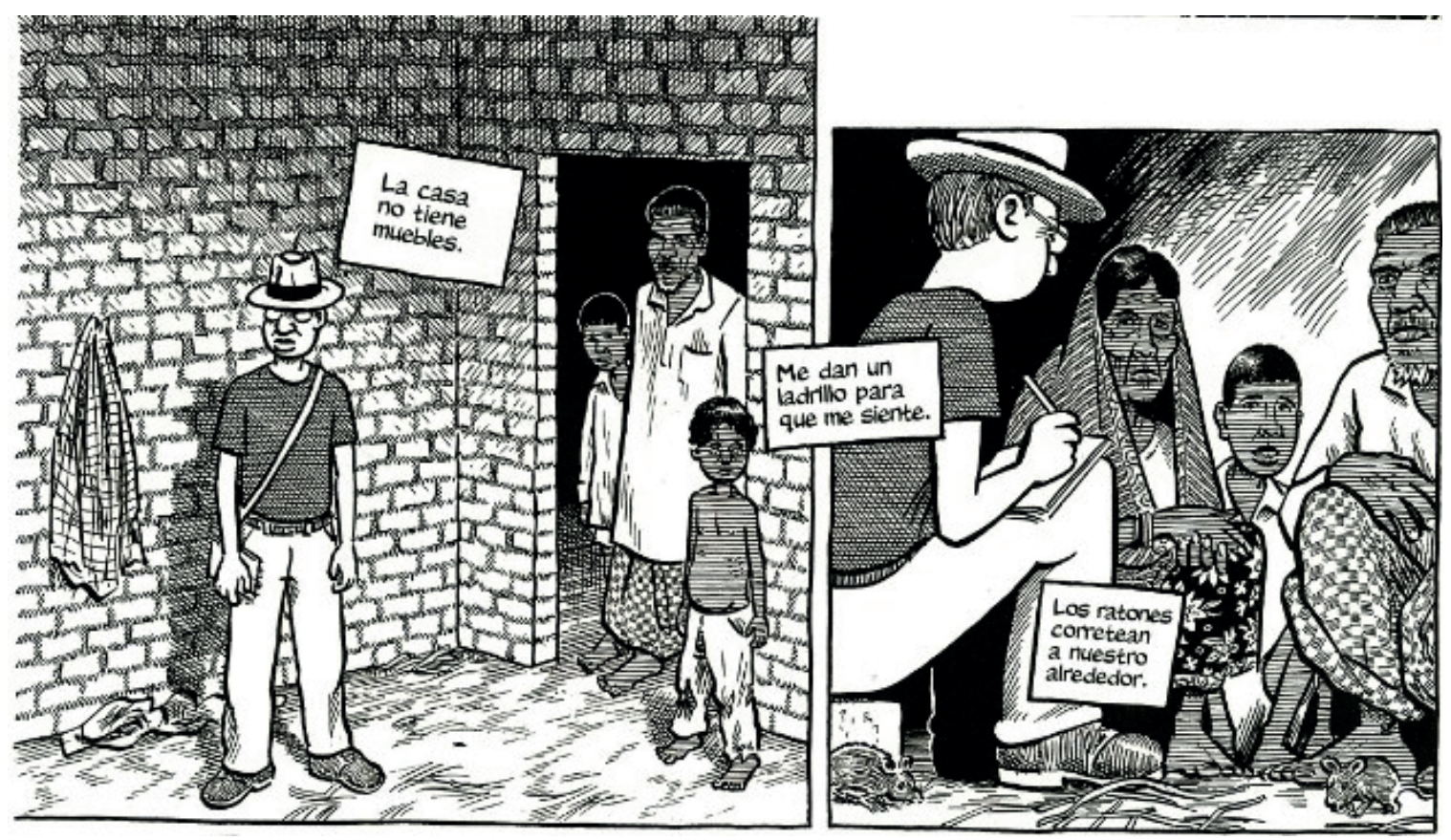

FIG. 6. Sacco, J. Reportajes. Barcelona, Reservoir Books, 2012. Joe Sacco en la India dando voz a los intocables. Viñetas de la página 173.

Siguiendo la estela de Sacco, muchos dibujantes han buscado encontrar grandes historias en lugares alejados del gran público occidental. Una de las propuestas más interesantes es la de Emmanuel Guibert con El fotógrafo ${ }^{29}$ —obra recientemente reeditada, aunque publicada en español ya en 2005-, quien trasladó al cómic las vivencias del fotógrafo Didier Lefèvre en la guerra de Afganistán de los años ochenta. Lefèvre es el protagonista de la obra y seguimos sus pasos para llegar a un puesto avanzado de Médicos Sin Fronteras, en el Afganistán que lucha contra la invasión soviética. Para dar aún mayor apariencia de objetividad y verosimilitud, el cómic combina el dibujo de Guibert con algunas de las más de cuatro mil fotografías que tomó Lefèvre. La lucha y la capacidad de adaptación del pueblo afgano y las dificultades que tienen los miembros de la ONG para ejercer su labor son los ejes del relato.

Otra propuesta tremendamente atractiva es la del italiano Igort. De antepasados ucranianos y muy interesado en la cultura rusa, Igort visitó Ucrania siguiendo los pasos del escritor ruso Antón Chéjov. Allí se dio cuenta de que mucha gente a la que entrevistaba tenía historias que contar sobre el pasado soviético y sobre los cambios que había provocado la caída de la URSS. Igort abandonó el proyecto de Chéjov y concibió Cuadernos ucranianos, ${ }^{30}$ que consistía en un potente trabajo gráfico — cómic, libro ilustrado, bocetos...- para recuperar la

\footnotetext{
${ }^{29}$ Guibert, Lefèvre, Lemercier. El fotógrafo. Madrid, Sins Entido, 2011.

${ }^{30}$ Igort. Cuadernos ucranianos. Memorias de los tiempos de la URSS. Madrid, Sins Entido, 2011.
} 

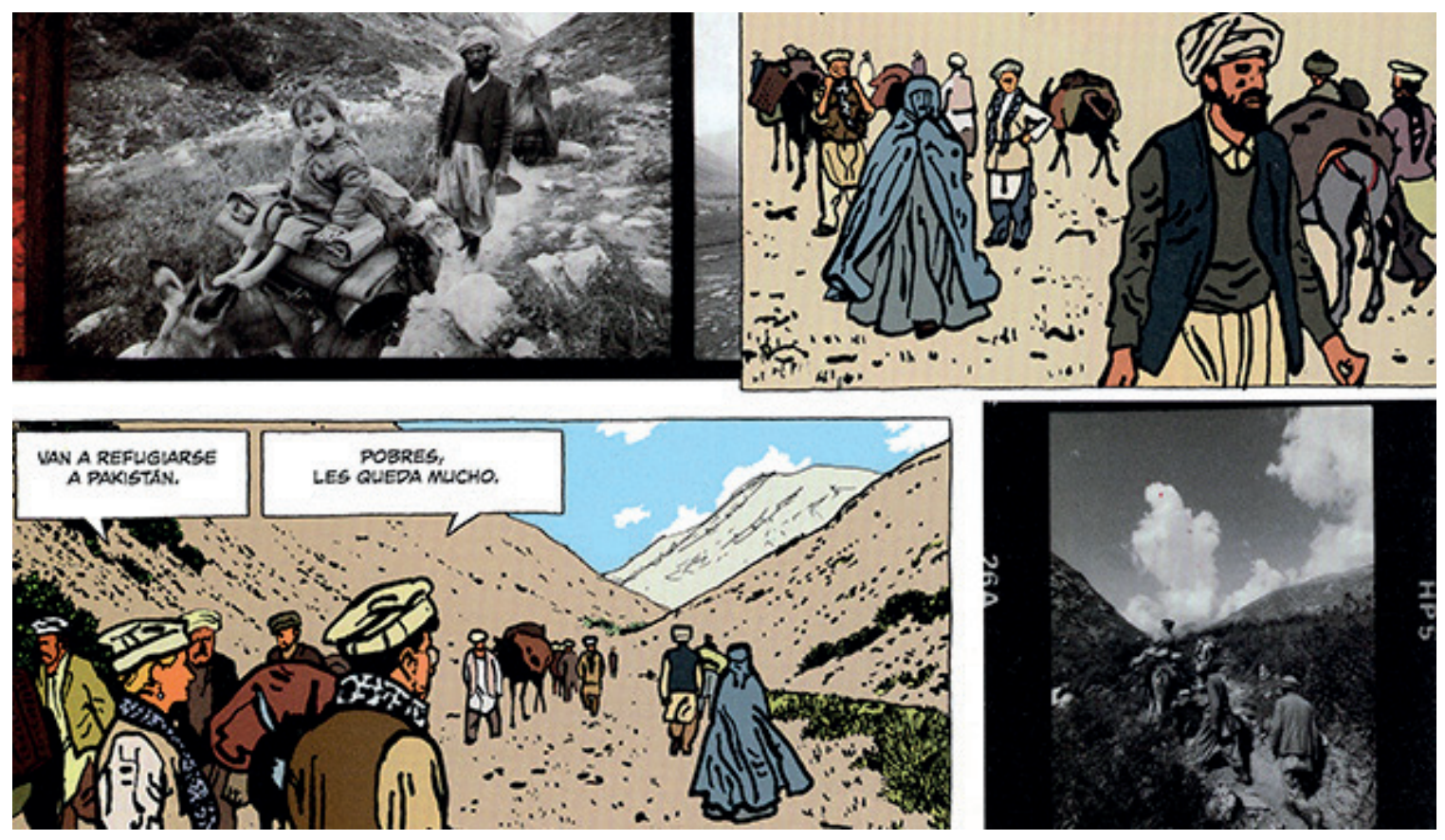

FIG 7. Guibert, Lefèvre, Lemercier. El fotógrafo. Madrid, Sins Entido, 2011. Viñetas de la página 52 en que se aprecia que la combinación de dibujo y fotografía es uno de sus elementos fundamentales.

memoria histórica de los tiempos más duros del estalinismo y del gran fracaso social que había supuesto la transición al capitalismo.

Tres años después, en 2014, Igort se acercó a uno de los temas más candentes de las Relaciones Internacionales del momento: la situación de la nueva Rusia de Vladimir Putin. Tras el asesinato en oscuras circunstancias de la periodista Anna Politkóvskaya, quien había estado investigando los crímenes del ejército ruso en Chechenia, el autor italiano creó Cuadernos rusos. ${ }^{31}$ En este cómic, que vuelve a combinar con elegancia las viñetas y las ilustraciones, Igort reconstruye el asesinato de la periodista y da a conocer lo que ella había descubierto sobre las actuaciones de los militares rusos en el Cáucaso.

Manteniendo la estructura del mencionado observador externo, Guy Delisle ha ido construyendo una narrativa muy original. El éxito de Pyongyang, ${ }^{32}$ en el que relataba su experiencia en Corea del Norte mientras trabajaba temporalmente en unos estudios de animación, permitió al autor canadiense ahondar en este tipo de propuestas. Hasta el momento ha publicado cuatro cómics que mantienen esta premisa: Guy Delisle viaja, por diferentes

\footnotetext{
${ }^{31}$ Igort. Cuadernos rusos. La guerra olvidada del Cáucaso. Barcelona, Salamandra Graphic, 2014.

32 Delisle, G. Pyongyang. Bilbao, Astiberri, 2005.
} 


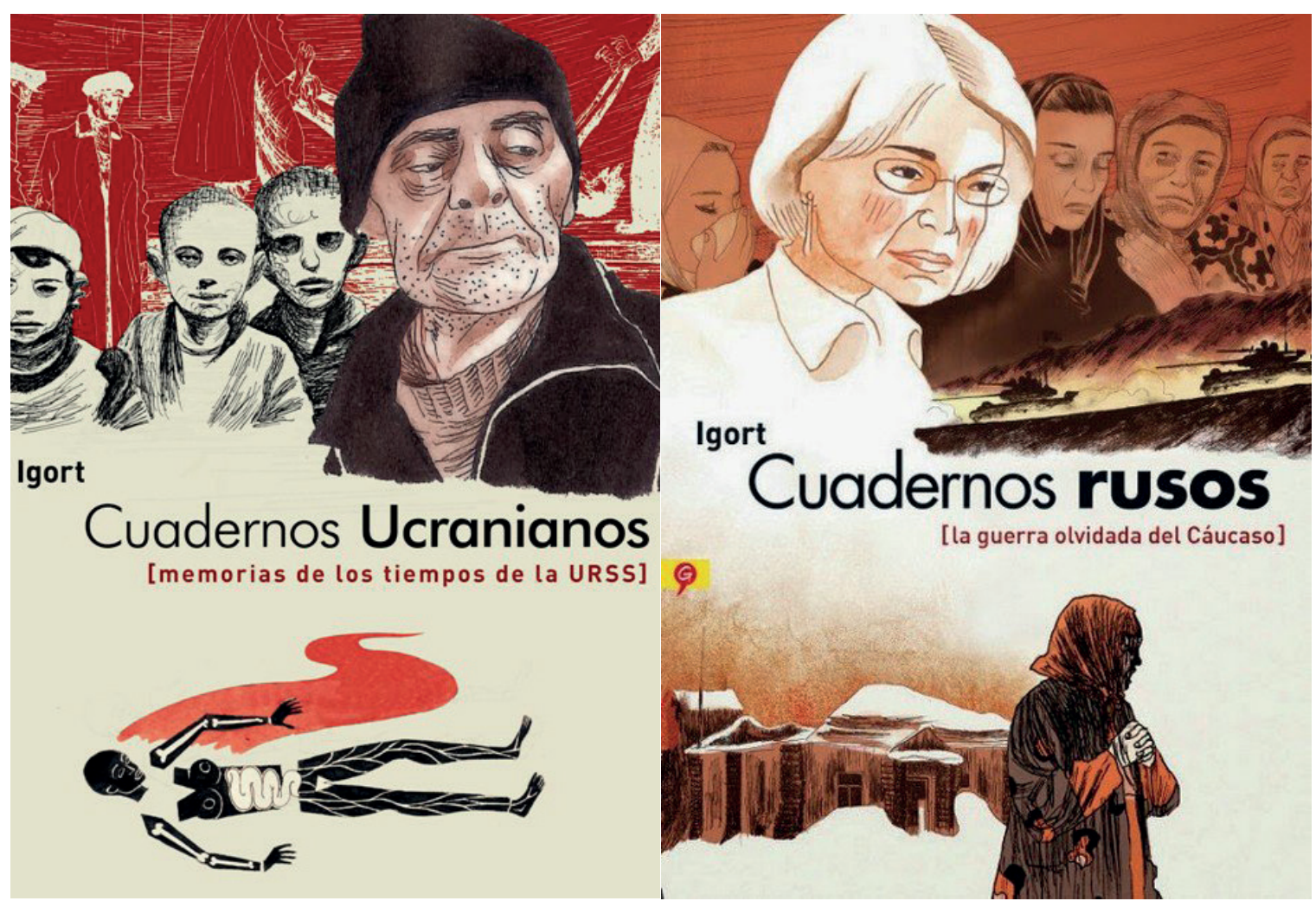

FIG. 8. Igort. Cuadernos ucranianos. Memorias de los tiempos de la URSS. Madrid, Sins Entido, 2011; y Cuadernos rusos. La guerra olvidada del Cáucaso. Barcelona, Salamandra Graphic, 2014. Portadas de los dos cómics del dibujante italiano sobre la Europa del Este.

motivos, a algún país que presenta un gran interés desde el punto de vista de la política internacional, y allí nos narra en primera persona sus experiencias cotidianas. Su capacidad de observación es muy elevada y mediante anécdotas y situaciones aparentemente intrascendentes, Delisle es capaz de analizar en profundidad las sociedades y los contextos con los que topa.

Aunque Pyongyang fue la obra que lo dio a conocer, el resto de cómics que siguen su esquema también rayan a gran altura. En Shenzen ${ }^{33}$ - que es anterior a Pyongyang- somos testigos del crecimiento descontrolado de una ciudad china, que en veinte años pasó de ser una pequeña urbe de provincias a convertirse en una auténtica megalópolis con millones de habitantes. Posteriormente, con Crónicas birmanas ${ }^{34}$ Delisle nos traslada al país del sudeste asiático, donde podemos observar la compleja situación política —una dictadura militar

\footnotetext{
33 Delisle, G. Shenzen. Bilbao, Astiberri, 2005.

${ }^{34}$ Delisle, G. Crónicas birmanas. Bilbao, Astiberri, 2008.
} 
de larga duración- y conocer la figura de Aung San Suu Kyi, la Premio Nobel de la Paz en 1991. Por último, su obra más reciente es Crónicas de Jerusalén, ${ }^{35}$ en la que el dibujante relata su vida en la Ciudad Santa mientras ejerce de padre. Sus encuentros con las consecuencias cotidianas del conflicto entre Israel y Palestina, sus visitas al muro o a lugares tan complejos como Hebrón, nos permiten conocer muchos elementos que generalmente pasan desapercibidos en los medios de comunicación. Todo ello, además, aderezado con un gran sentido del humor.
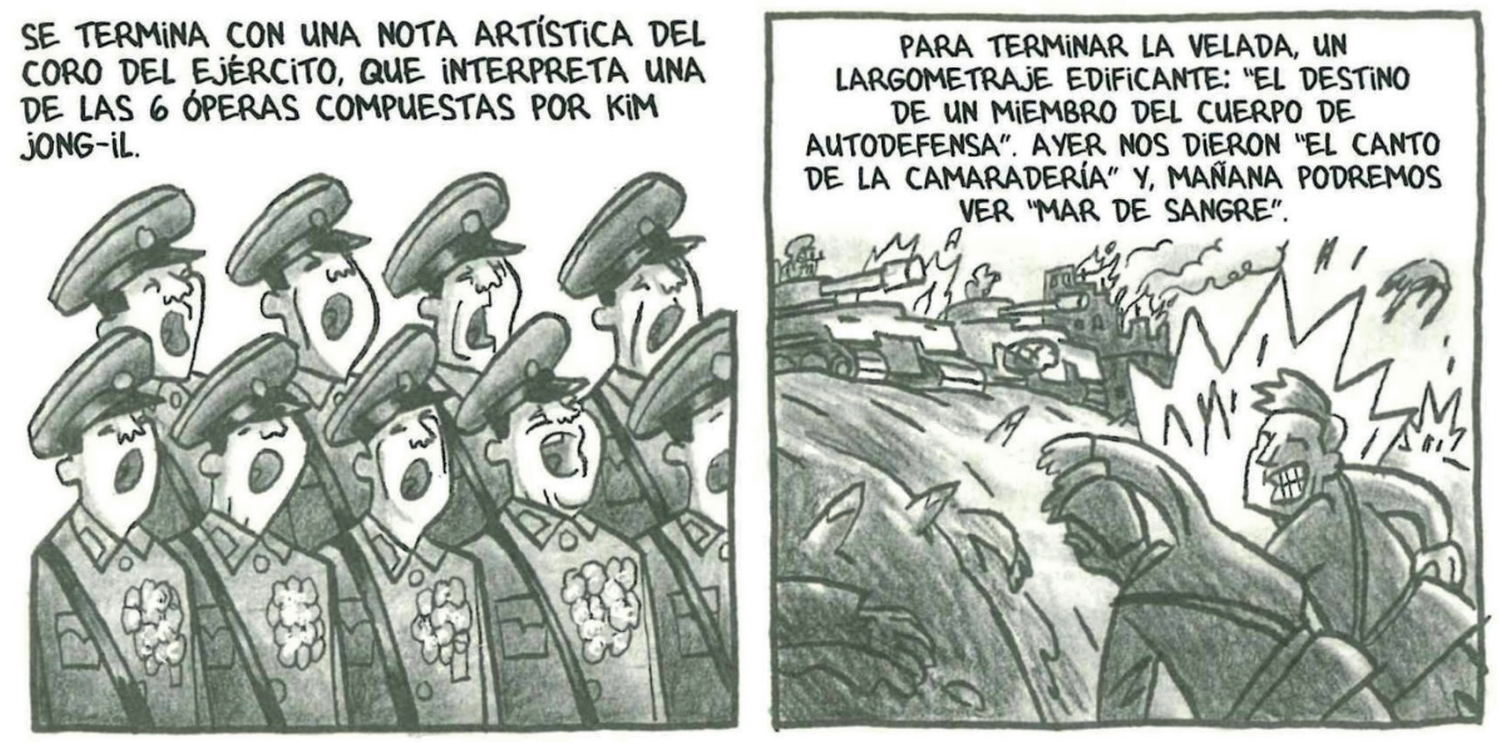

FIG. 9. Delisle, G. Pyongyang. Bilbao, Astiberri, 2005. Viñetas de la página 147 en que Guy Delisle narra una de sus veladas cotidianas en Corea del Norte.

Sin dejar el conflicto árabe-israelí, otro cómic con una perspectiva llamativa es Una judía americana perdida en Israel, ${ }^{36}$ de Sarah Glidden. La dibujante estadounidense viajó a Israel en el verano de 2007 gracias al programa Birthright Israel — derecho de nacimiento-, que permite visitar Israel con los gastos pagados a todos los judíos del mundo. Lo más interesante del cómic es la evolución de Glidden y sus reacciones cuando va descubriendo la realidad del conflicto y la propaganda que lleva a cabo el Estado de Israel.

Para acabar, y ante la imposibilidad de llevar a cabo un recorrido exhaustivo por todas las obras que utilizan al observador externo para representar las realidades de lugares más o menos exóticos, tan solo mencionar algunas obras que merecen nuestra atención:

- Kabul disco, de Nicolas Wild (Ponent Mon, 2008), que presenta la situación de Afganistán tras el final de la guerra iniciada por George Bush.

\footnotetext{
35 Delisle, G. Crónicas de Jerusalén. Bilbao, Astiberri, 2011.

${ }^{36}$ Glidden, S. Una judía americana perdida en Israel. Barcelona, Norma Editorial, 2011.
} 
- Macedonia, de Harvey Pekar y Heather Roberson (La Cúpula, 2008), que analiza el proceso de transición macedonio y se centra en los aspectos relacionados con la legislación y los derechos humanos.

- El coche de Intisar, de Pedro Riera y Nacho Casanova (Glénat, 2011), que retrata la situación de la mujer en Yemen.

- Chernóbil. La zona, de Natacha Bustos y Francisco Sánchez (Glénat, 2011), que estudia lo que sucedió en la central nuclear de Chernóbil y sus consecuencias para los habitantes de Pripyat.

\subsection{Humorístico}

Por último, la tercera categoría en la que he dividido los cómics que tratan las relaciones internacionales es aquella cuya característica principal es el tono humorístico. Algunas de las obras anteriores, pienso especialmente en las de Sattouf, Satrapi o Delisle, tienen un gran componente satírico, pero ese no es su objetivo principal. En cambio, la obra que presentaré a continuación, pese a tratar temas que podríamos denominar serios, busca continuamente la carcajada del lector.

En primer lugar, Quai d'Orsay ${ }^{37}$ es, quizás junto con los ensayos históricos del primer apartado, el cómic más puramente enfocado en las relaciones internacionales. De la mano de Arthur Vlaminck, un brillante joven que es contratado en el ministerio de Asuntos Exteriores francés, vemos desde dentro el funcionamiento de la diplomacia internacional. El estrafalario ministro, inspirado en Dominique de Villepin, tiene una obsesión con los lenguajes y Vlaminck y el resto de asesores serán las víctimas constantes de sus actitudes neuróticas.

Siempre en el marco de situaciones muy divertidas y con la expresividad y el dinamismo del dibujo de Blain, somos testigos del funcionamiento de un ministerio, asistimos a reuniones de la ONU o de la Unión Europea, viajamos en visitas oficiales... Pocas maneras mejores para acercarnos a las interioridades de la política internacional: enfrentamientos diplomáticos entre grandes potencias, negociaciones muy duras para conseguir victorias pírricas, decisiones controvertidas y la búsqueda del maquillaje idóneo ante la opinión pública, relaciones con dictaduras y conflictos de intereses de todo tipo tienen cabida en Quai d'Orsay.

\section{Conclusión}

El cómic ha ganado respetabilidad cultural en los últimos años y ha demostrado ser capaz de tratar con profundidad cualquier tema, por complejo que este sea. En el caso de las

37 Lanzac, A. y Blain, C. Quai d'orsay. Crónicas diplomáticas. Barcelona, Norma Editorial, 2011 y 2012. Edición en dos volúmenes. 
relaciones internacionales, disciplina en auge en las últimas décadas, el cómic ha probado en repetidas ocasiones que es muy efectivo como puerta de entrada a la realidad sociopolítica de cualquier país.

Mediante diferentes estrategias, multitud de autores y autoras han conseguido que abramos los ojos a conflictos y situaciones generalmente desconocidas para el gran público. La combinación del dibujo, que permite introducirnos en el contexto de manera mucho más efectiva que en un ensayo; el valor del testimonio personal del autor, mucho más trabajado que las piezas frecuentemente enlatadas de las grandes agencias de noticias; y la humanización de los habitantes de estos lugares, que en la mayoría de los cómics analizados tienen un papel destacado, permiten dotar al cómic de unas cualidades que no se encuentran en otros medios.

En definitiva, el cómic y las relaciones internacionales han demostrado ser una pareja que se entiende muy bien y todo hace indicar que tendrán un brillante futuro en común. Pese a los agoreros que hablaban del final de la Historia, ${ }^{38}$ esta demuestra que está muy viva y el cómic seguirá dispuesto a tratar los acontecimientos actuales y futuros. Ya lo sabéis, no hay mejor manera de conocer un conflicto o un país que leyendo un buen cómic sobre él.

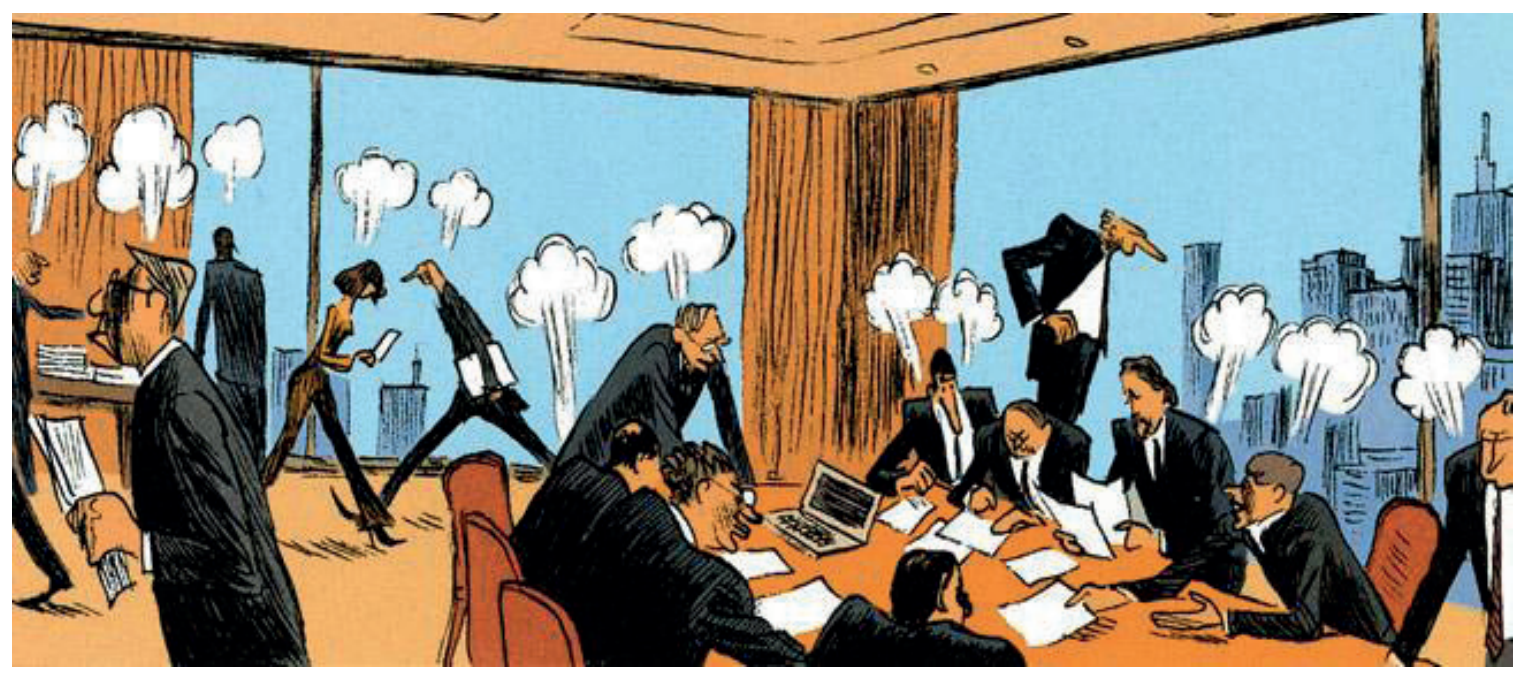

FIG. 10. Lanzac, A. y Blain, C. Quai d'orsay. Crónicas diplomáticas. Barcelona, Norma Editorial, 2012. Viñeta de la página 11 del segundo tomo, en la que se observa como el dibujo de Christophe Blain consigue recrear con gran viveza la tensión cotidiana de los asesores del ministro.

\footnotetext{
${ }^{38}$ Véase la introducción del artículo donde las ideas de Francis Fukuyama, que auguraba el fin de la Historia tras el final de la Guerra Fría, son desacreditadas por la realidad de los años noventa y el inicio del siglo xxI.
} 